\title{
Ophthalmic Manifestations and Type 4 of Hypersensitivity of Steven Johnson Syndrome-Toxic Epidermal Necrosis in Infants-Management and Literature Review
}

\author{
Juna Musa'*, Blina Abdullahu' ${ }^{2}$, Ali Guy ${ }^{3}$, Luela Hallunovaj ${ }^{4}$, Amro \\ Elrefaei $^{5}$, Benan Barakat ${ }^{6}$, Ina Kola ${ }^{7}$, Ermira Kola ${ }^{2}$, Holgena Cepani ${ }^{8}$, \\ Inva Mamica ${ }^{9}$ and Alketa Tandili ${ }^{9}$ \\ ${ }^{1}$ Department of Surgery, Critical Care and Trauma, Mayo Clinic, Rochester, Minnesota, USA \\ ${ }^{2}$ University Hospital, "Mother Teresa”, Tirana, Albania \\ ${ }^{3}$ Clinical Assistant Professor, Department of Physical Medicine and Rehabilitation, New York \\ University School of Medicine, NYU Medical Center, New York, USA \\ ${ }^{4}$ Ophthalmologist, Eye Clinic UHC, "Mother Teresa", Tirana, Albania \\ ${ }^{5}$ Division of MFM, Department of Obstetrics and Gynecology, Rochester, Minnesota, USA \\ ${ }^{6}$ Department of Neurology, Division of Multiple Sclerosis and Autoimmune Neurology, Mayo \\ Clinic, Rochester, Minnesota, USA \\ ${ }^{7}$ Department of Burns and Plastic Surgery, Tirana, Albania \\ ${ }^{8}$ Ophthalmologist, Eye Clinic UHC, "Mother Teresa", Tirana, Albania \\ ${ }^{9}$ Mother Teresa Hospital, Tirana, Albania \\ *Corresponding Author: Juna Musa, Department of Surgery, Critical Care and \\ Trauma, Mayo Clinic, Rochester, Minnesota, USA.
}

Received: August 17, 2020

Published: September 17, 2020

(C) All rights are reserved by Juna Musa., et al.

\begin{abstract}
Steven-Johnson syndrome (SJS) is a rare and sometimes life-threatening type IV (subtype C) hypersensitivity reaction. It is usually triggered by a reaction to a medication, but the exact etiology is unclear. However, around half of cases are idiopathic. Clinical features in SJS start with a prodrome of unspecific symptoms (flu-like symptoms), followed by lesions of the skin, eye and mucous membranes of oral, gastrointestinal, respiratory and gynecologic tissues.

There is no definite effective systemic and local treatment for SJS therefore; most patients with Stevens-Johnson syndrome are treated symptomatically. Early detection, the withdrawal of the suspect drug and aggressive treatment are crucial.

Besides the systemic support, an eye examination should be an essential component of the evaluation of any patient with suspected Syndrome to decrease the risk of long-term ocular compromise. Stevens-Johnson syndrome can cause eye inflammation, dry eye, trichiasis and light sensitivity. In severe cases, ocular complications can lead to blindness.

We report the case of a four-year-old girl with SJS presenting with erythematous vesicular lesions, purulent tonsillitis and ocular manifestations like bilateral conjunctivitis. The case came as a referral to the ophthalmology department after getting the proper treatment for the acute phase with steroids, human Ig, eye drops and antibiotics. Along with primarily supportive and symptomatic treatment, we emphasize the importance of eye evaluation often neglected by the life-threatening concerns to ensure a better prognosis for the child in the long term.

Keywords: Stevens-Johnson Syndrome; TEN-Life-Threatening Skin Emergencies; Mucous Membranes; Ocular Manifestations; Trichiasis
\end{abstract}

Citation: Juna Musa., et al. "Ophthalmic Manifestations and Type 4 of Hypersensitivity of Steven Johnson Syndrome-Toxic Epidermal Necrosis in Infants-Management and Literature Review". Acta Scientific Medical Sciences 4.10 (2020): 50-53. 


\section{Introduction}

Steven-Johnson syndrome is a serious systemic disorder with the potential to cause fatal results. It is a rare with disease, with an approximate incidence of 2-7 cases per million people in one year. SJS affects mostly children and young adults, however, it can also affects more the children and young adults, but can affect all the ages. Females are more affected than men. SJS is a type IV (subtype C) hypersensitivity reaction that involves the skin and the mucous membranes. It starts with flu-like symptoms, followed by a painful rash that spreads and blisters, then the affected skin dies, sheds and begins to heal after several days. May present with significant involvement of ocular, oral, nasal, urethral, gastrointestinal and lower respiratory tract mucous membranes. Severe complications can occur as shock, organ failure, acute respiratory failure, visual impairment and blindness. The ocular problems affect $60 \%$ of patients with Steven-Johnson syndrome and they appear in the form of conjunctivitis, scarring of the conjunctiva, inflammation inside the eye, corneal blisters and perforation. Ocular damage is more frequent in patients with epidermal detachment $>10 \%$ of the total body surface area. Steven-Johnson is a medical emergency and requires hospitalization. SJS is caused by an adverse reaction to certain medications as antibacterial sulfonamides, pain reliever, allopurinol, anticonvulsants, antipsychotics. Also, infections as mycoplasma pneumonia, vaccinations can trigger the development of SJS. Certain genetic variations, a weakened immune system, an HIV infection, a previous history and a family history of this syndrome can predispose and increase the risk. Genetic testing before taking some drugs is important to prevent this syndrome [1,2].

\section{Case Presentation}

A 4 year old female patient presents to the Pediatric Emergency Department with a history of bilateral conjunctivitis and erythematous vesicular lesions. The latter primarily localized in the palms of the hands and soles of the foot to further being disseminated in all the skin area as well as in the mucosa. The overall skin and mucosa involvement started 3 days prior to presenting in the ER department following a recent history of acute stomato-glossitis and Ibuprofen intake to dominate the mild pain.

Patient was subsequently admitted to the critical care unit at the Mother Teresa Hospital where the Steven-Johnson syndrome was confirmed by biopsy.
Following the acute phase the patient presents with aggravated symptoms of eye involvement, therefore specialized treatment was applied upon consultation with ophthalmology department.

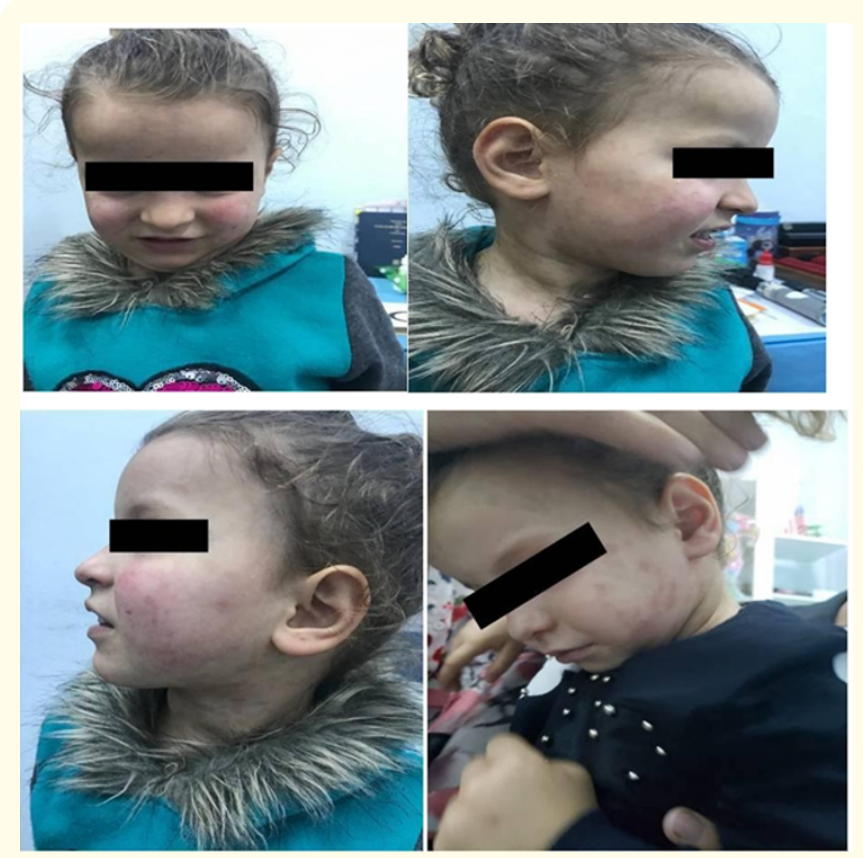

Figure 1: Patient presents to the ER with erythematous vesicular rashes located on the face, neck and trunk.

\section{Physical examination}

Upon admission the general condition appears to be altered with the patient experiencing malaise but an overall alert condition. There are pathognomonic target skin lesion with a vesicular core lesion surrounded by macular erythema symmetrically distributed on the face, trunk as well as limb areas with covering less than $10 \%$ of the body's surface area. Exfoliation of the outermost epidermal layer was noted upon rubbing of the skin (positive Nikolsky's sign).

The mother referred that patient couldn't swallow properly and a careful examination of oral cavity revealed enlarged, hyperemic tonsils with bilateral pus-filled spots.

Further during observation, infected crusts appear in the upper and lower lip. There is also eye involvement with bilateral conjunctivitis with clear, watery discharge. 
Laboratory assessment showed an elevated Erythrocyte Sedimentation Rate of $25 \mathrm{~mm} /$ hour and otherwise no significant change in complete blood count and biochemical analysis.

They were administrated saline infusion, intravenous human immunoglobulin and were started oral antibiotic therapy with ceftriaxone and vancomycin as well as glucocorticoid therapy with prednisolone and methylprednisolone. Furthermore, tobrex and lacrinorm were applied topically for the conjunctivitis and the watery secretions.

Given the considerable ocular involvement after the acute phase with photophobia, increased epiphora and hyperemia there was consulted with the ophthalmology department. After further examination there were also trichiasis in upper palpebrae, inferolateral deepithelization without symblepharon present.

Therefore, mechanical epilation with forceps as a temporary measure were applied. Also, intensive lubrication with lacrinorm gel and Vitan and topical profilactic antibiotic therapy with vigamox were applied as a measure to prevent infection.

Eye lens were intended but patient was not cooperative given the young age.

\section{Diagnosis}

There are several factors that contribute to a proper diagnosis: a detailed patient history, characteristic symptoms, in-depth clinical evaluation and the gold standard of skin biopsy. Manifestations of Steven-Johnson syndrome include purpuric macules, bullae formation and targetoid lesions. Furthermore, there is necrosis affecting the epidermal and dermal layer of the skin and also the membrane lining of mucosa causing them to detach. Involvement of $10 \%$ of body surface indicate a diagnosis of Steven-Johnson syndrome while a more than $30 \%$ involvement is associated with a more severe form of this dermatologic disorder where mortality rates can approach $40 \%$. Nikolsky's sign, demonstrated when mild pressure is applied on the border of an intact blister resulting in dislodgment of upper layer skin from lower layers is a hallmark in clinical differential diagnosis from pemphigus vulgaris and erythema multiforme.

Furthermore, a skin biopsy from affected skin reveals subepidermal blistering, necrotic tissue and thickened epithelial tissue, typical features in SJS.

\section{Discussion}

SJS is a rare, immune mediated disorder of the skin considered a medical emergency which usually requires hospitalization. Mucosal involvement is very common, affecting $90 \%$ of patients. Because ocular involvement is inevitable, presenting in more than $80 \%$ of patients with SJS, evaluation by an ophthalmologist should be done urgently. The spectrum of SJS with ocular manifestations can range from acute to chronic, if left unattended [1]. Acute phases begin with bilateral conjunctivitis and loss of surface epithelium, chronic phases show symptoms of chronic ocular inflammation and severe dry eye. Early detection, prompt and adequate treatment are highly recommended to prevent symblepharon (partial or complete adhesion of the palpebral conjunctiva of the eyelid to the bulbar conjunctiva of the eyeball), eyelid malabsorption, dry eye, corneal disease and irreversible changes [2]. Usually, the acute phase has an onset of 8 - 12 days.

Management of SJS had been growing over the past years with rising usage of early amniotic membrane transplantation to treat severe ocular manifestations [3]. Gregory., et al. [4] in his case series suggested an acute phase grading system to be followed when AMT is required as a line of treatment. He suggested that to preserve vision and minimize the ocular scarring hazard and dry eyes, AMT should be used if there is corneal epithelial defect beyond punctate keratopathy, or 1 lid margin at least with stain including 1 $\mathrm{cm}$ or more in largest diameter [4]. The use of IVIG was debatable [5]. It did not show any decrease in severity of significant ocular complications as Yip., et al. [6] mentioned in his study. But, combination of IVIG with corticosteroids was beneficial in decreasing severity of the disease and improving ophthalmic lesions as mentioned in Aihara., et al [7].

Our 4 year old patient presented to the ER during the acute stage with altered mental status, erythematous skin lesions and bilateral conjunctivitis. Systemic treatment with ceftriaxone and vancomycin, steroids, human Ig and IV boluses of normal saline and dextrose were administered. 3 days later she presented to the ophthalmologist for further evaluation and underwent a wide range of treatments. Manual removal of eyelashes given she had trichiasis, lubrification with lacrinorm gel and antibiotic prophylaxis (Vigamox) due to corneal infiltration. Tropicamide eye drops were also used. Lastly, effort was made to keep in place contact lenses in which the patient could not do because of increased irritation, 
which in the long run is probably a good thing given the fact the chronic use can eventually lead to dry eyes. For symblepharon, in which our patient did not manifest, Amniotic membrane transplant (AMT) is considered as treatment of choice as well as ocular surface reconstruction surgery [8].

\section{Conclusion}

Several cases of Steven Johnson syndrome and Toxic Epidermal Necrosis have been reported. It is recommended that all patients admitted for SJS/TEN or suspicious of SJS/TEN should be evaluated and then closely followed by an ophthalmologist due to high risk of progressing of the disease and invasive visual intervention risk. The diagnose made in time and right treatment prevent a possible

\section{Bibliography}

1. Stevens-Johnson Syndrome - EyeWiki (2018).

2. Ocular Manifestations of Stevens-Johnson Syndrome and Their Management (2016).

3. Chow LLW., et al. "Comparison of the acute ocular manifestations of Stevens-Johnson syndrome and toxic epidermal necrolysis in Chinese eyes: a 15-year retrospective study". BMC Ophthalmology 17.65 (2017).

4. Gregory DG. "New grading system and treatment guidelines for the acute ocular manifestations of Stevens-Johnson syndrome". Ophthalmology 123.8 (2016): 1653-1658.

5. Kohanim S., et al. "Stevens-Johnson syndrome/toxic epidermal necrolysis - a comprehensive review and guide to therapy. I. Systemic disease". The Ocular Surface 14.1 (2016): 2-19.

6. Yip LW., et al. "High-dose intravenous immunoglobulin in the treatment of toxic epidermal necrolysis: a study of ocular benefits". Eye 19.8 (2005): 846-853.

7. Aihara M., et al. "Efficacy of additional i.v. immunoglobulin to steroid therapy in Stevens-Johnson syndrome and toxic epidermal necrolysis". Journal of Dermatological 42.8 (2015): 768-777.

8. Amniotic membrane transplantation can be useful in symblepharon lysis surgery (2010).

\section{Assets from publication with us}

- Prompt Acknowledgement after receiving the article

- Thorough Double blinded peer review

- Rapid Publication

- Issue of Publication Certificate

- High visibility of your Published work

Website: $\underline{\text { www.actascientific.com/ }}$

Submit Article: www.actascientific.com/submission.php

Email us: editor@actascientific.com

Contact us: +919182824667 